\title{
On individual subsequential ergodic theorem in von Neumann algebras
}

\author{
by \\ Semyon Litvinov (Fargo, ND) and Farrukh Mukhamedov (Tashkent)
}

\begin{abstract}
We use a non-commutative generalization of the Banach Principle to show that the classical individual ergodic theorem for subsequences generated by means of uniform sequences can be extended to the von Neumann algebra setting.
\end{abstract}

0. Introduction. The study of almost everywhere convergence of the ergodic averages in the non-commutative setting was initiated by a number of authors among whom we mention Lance [5] and Yeadon [11]. Individual ergodic theorems have been established for algebras with states as well as for algebras equipped with a semifinite trace. The study of almost everywhere convergence of weighted and subsequential averages in von Neumann algebras is relatively new. So far, not much is known in this direction. Recently, a non-commutative analog of the classical Banach Principle, on convergence of sequences of measurable functions generated by a sequence of linear maps on $L^{p}$-spaces, was established in [3]. It is expected that, as in the commutative case, this principle will be instrumental in obtaining various convergence results for the averages in non-commutative setting. In [8], an individual ergodic theorem for subsequences was proved, where the proof was based on application of the "commutative" Banach Principle. In this paper we use the ergodic theorem of Yeadon [11] together with the results of [3], adjusted to the bilateral almost uniform convergence, to show that the main result of [8] also holds in the vNa setting.

1. Preliminaries. Let $M$ be a von Neumann algebra (vNa) acting on a Hilbert space $H$. Let $I$ be the unit of $M$, and let $\tau$ be a faithful normal semifinite trace on $M$. Denote by $P(M)$ the complete lattice of all projections in $M$. Let $A(M)$ be the set of all closed operators affiliated with $M$. An operator $x \in A(M)$ is said to be $\tau$-measurable if for each $\varepsilon>0$ there

2000 Mathematics Subject Classification: Primary 46L50; Secondary 47A35.

Supported by ND EPSCoR through NSF grant \#OSR-9452892. 
exists $e \in P(M)$ such that $\tau\left(e^{\perp}\right) \leq \varepsilon$ and $e H \subset D_{x}$. Let $\bar{M}$ be the set of all $\tau$-measurable operators with the measure topology given by, say, the following system of neighborhoods of zero: for $\varepsilon>0, \delta>0$,

$$
V(\varepsilon, \delta)=\left\{x \in \bar{M}:\|x e\| \leq \varepsilon \text { for some } e \in P(M) \text { with } \tau\left(e^{\perp}\right) \leq \delta\right\},
$$

where $\|\cdot\|$ is the operator norm in $M$. For a positive self-adjoint operator $x=\int_{0}^{\infty} \lambda d e_{\lambda}$ affiliated with $M$ one can define

$$
\tau(x)=\sup _{n} \tau\left(\int_{0}^{n} \lambda d e_{\lambda}\right)=\int_{0}^{\infty} d \tau\left(e_{\lambda}\right) .
$$

For $0<p \leq \infty$, let

$$
L^{p}=L^{p}(M, \tau)=\left\{x \in A(M):\|x\|_{p}=\tau\left(\left(\left(x^{*} x\right)^{1 / 2}\right)^{p}\right)^{1 / p}<\infty\right\}
$$

if $p \neq \infty$, and let $L^{\infty}=M$. For properties of non-commutative $L^{p}$-spaces which are naturally imbedded in $\bar{M}$, see $[2,6,9,10]$. There are many different types of convergence in $\bar{M}$ each of which, in the commutative case with finite measure, reduces to the usual almost everywhere convergence. We deal with the so-called bilateral almost uniform (b.a.u.) convergence in $\bar{M}$ for which $a_{n} \rightarrow \widehat{a}$ means that for every $\varepsilon>0$ there exists $p \in P(M)$ with $\tau\left(p^{\perp}\right) \leq \varepsilon$ such that $\left\|p\left(a_{n}-\widehat{a}\right) p\right\| \rightarrow 0$.

The following is a non-commutative variant of the Banach Principle [3] adapted to the b.a.u. convergence.

THEOREM 1. Let $M$ be a vNa with unit $I$ and with a faithful normal semifinite trace $\tau$. Let $\bar{M}$ be the topological $*$-algebra of all $\tau$-measurable operators. For a Banach space $X$, assume that $a_{n}: X \rightarrow \bar{M}$ is a sequence of continuous linear maps satisfying the condition:

(i) for every $x \in X$ and $\varepsilon>0$ there is an operator $b \in M, 0 \leq b \leq I$, with $\tau(I-b) \leq \varepsilon$ such that $\sup _{n}\left\{\left\|b a_{n}(x) b\right\|\right\}<\infty$.

If, for every $x$ from a dense subset $X_{0} \subset X$,

(ii) $a_{n}(x)$ converges b.a.u. in $\bar{M}$, then (ii) holds on all $X$.

Sketch of proof (for more details see [3]). (I) It is easy to see that, given $x \in X, p \in P(M)$ with $\tau(p)<\infty$ and $\varepsilon, \delta>0$ it is enough to find a projection $q \leq p$ such that $\tau(p-q)<\varepsilon$ and

$$
\left\|q\left(a_{m}(x)-a_{n}(x)\right) q\right\|<\delta
$$

for any $m, n \geq N(\varepsilon, \delta, p)$. In its turn, this inequality will follow if we construct a sequence $\left\{y_{k}\right\} \subset X$ and a projection $g \in P(M)$ with $g \leq p$ and $\tau(p-g) \leq \varepsilon / 2$ enjoying the properties

(a) $x+y_{k} \in X_{0}$ for all $k$ and 
(b) $\left\|g a_{n}\left(y_{k}\right) g\right\| \rightarrow 0$ uniformly in $n$.

(II) We define, for every pair $L$ and $k$ of positive integers, the set $X_{L, k}=\left\{x \in X: \exists 0 \leq b \leq p\right.$ with $\tau(p-b) \leq \varepsilon / 2^{k+3}$,

$$
\left.\sup _{n}\left\{\left\|b a_{n}(x) b\right\|\right\} \leq L\right\} .
$$

and show that $X_{L, k}$ is closed in $X$.

(III) Next, since the condition (i) implies that

$$
X=\bigcup_{L=1}^{\infty} X_{L, k},
$$

by the Baire category theorem we find $L_{k}, x_{k} \in X$ and $\delta_{k}>0$ such that for every $x \in X$ satisfying $\left\|x-x_{k}\right\|_{X}<\delta_{k}$ there exists an operator $0 \leq b_{x, k} \leq p$ with $\tau\left(p-b_{x, k}\right) \leq \varepsilon / 2^{k+3}$ for which

$$
\sup _{n}\left\{\left\|b_{x, k} a_{n}(x) b_{x, k}\right\|\right\} \leq L_{k} .
$$

(IV) If $f_{x, k}$ is the spectral projection of $b_{x, k}$ in the von Neumann algebra $p M p$ corresponding to the interval $[1 / 2,1]$, then $\tau\left(p-f_{x, k}\right) \leq \varepsilon / 2^{k+2}$ and $\sup _{n}\left\{\left\|f_{x, k} a_{n}(x) f_{x, k}\right\|\right\} \leq 4 L_{k}$. If $\left\|x-x_{k}\right\|_{X}<\delta_{k}$ and $g_{x, k}=f_{x, k} \wedge f_{x_{k}, k}$, then $\tau\left(p-g_{x, k}\right) \leq \varepsilon / 2^{k+1}$ and $\sup _{n}\left\{\left\|g_{x, k} a_{n}\left(x-x_{k}\right) g_{x, k}\right\|\right\} \leq 8 L_{k}$. This means that if $\gamma_{k}=\delta_{k} /\left(8 L_{k}\right)$, then $\|z\|_{X} \leq \gamma_{k}$ entails the existence of $g_{z, k} \in P(M)$, $0 \leq g_{z, k} \leq p$, with $\tau\left(p-g_{z, k}\right) \leq \varepsilon / 2^{k+1}$ such that $\sup _{n}\left\{\left\|g_{z, k} a_{n}(z) g_{z, k}\right\|\right\} \leq 1$. If $g=\bigwedge_{k=1}^{\infty} g_{z, k}$, then $\tau(p-g) \leq \varepsilon / 2$ and $\|z\|_{X}<\gamma_{k}$ implies

$$
\sup _{n}\left\{\left\|g a_{n}(z) g\right\|\right\} \leq 1 \text {. }
$$

(V) Since $X_{0}$ is dense in $X$, for every $k$, there is a $z_{k} \in X$ satisfying $\left\|z_{k}\right\|_{X}<\gamma_{k}$ for which the condition (a) holds with $y_{k}=z_{k} / k$. It remains to note that (b) follows from the definition of $\left\{y_{k}\right\}$.

A positive linear map $\alpha: L^{1} \rightarrow L^{1}$ will be called an absolute contraction if $\alpha(I) \leq I$ and $\tau(\alpha(x)) \leq \tau(x)$ for every $x \geq 0$. In [11], the following form of non-commutative individual ergodic theorem was proved.

THEOREM 2. If $\alpha$ is an absolute contraction in $L^{1}=L^{1}(M, \tau)$, then, for every $x \in L^{1}$, the averages

$$
a_{n}(x)=\frac{1}{n} \sum_{k=0}^{n-1} \alpha^{k}(x)
$$

converge b.a.u. in $L^{1}$.

The key role in the proof of Theorem 2 is played by the so-called maximal ergodic theorem: 
Theorem 3 (see [11]). Let $L^{1}$, $\alpha$ be as above. Then for every $x \in L^{1}$ and $\varepsilon>0$ there exists $p \in P(M)$ with $\tau\left(p^{\perp}\right) \leq 4 \varepsilon^{-1}\|x\|_{1}$ such that

$$
\left\|p a_{n}(x) p\right\| \leq 4 \varepsilon \quad \text { for every } n .
$$

In order to illustrate how the non-commutative Banach Principle may work, we derive Theorem 2 from Theorem 3. First, note that $\left\{a_{n}(\cdot)\right\}$ is a sequence of positive linear maps from a Banach space $X=L^{1}$ to $L^{1} \subset$ $\bar{M}$ which are continuous under $\|\cdot\|_{1}$, hence as maps from $L^{1}$ to $\bar{M}$. The condition (i) of Theorem 1 is satisfied via Theorem 3, so it remains to find a dense subset $X_{0}$ in $L^{1}$ on which the b.a.u. convergence would take place. By Kadison's inequality [4], for every $x \in L^{1} \cap L^{2} \cap M_{s}$,

$$
\|\alpha(x)\|_{2}^{2}=\tau\left(\alpha(x)^{2}\right) \leq \tau\left(\alpha\left(x^{2}\right)\right) \leq \tau\left(x^{2}\right)=\|x\|_{2}^{2},
$$

i.e. $\alpha$ (after its unique extension) is a contraction in the real Hilbert space $H=H_{\tau}$, the completion of $L^{1} \cap L^{2} \cap M_{s}$ with respect to the norm $\|\cdot\|_{2}$ (induced by the inner product $\left.(x, y)=\tau(x y), x, y \in L^{2} \cap M_{s}\right)$. Applying a standard argument, we show that the set

$$
H_{0}=\left\{x+(y-\alpha(y)): x \in H \text { with } \alpha(x)=x, y \in M_{s}\right\}
$$

is dense in $H$. Indeed, it is enough to check that $x \perp(y-\alpha(y))$ with $x \in H$ and $y \in M_{s}$ would imply $\alpha(x)=x$. We have $0=(x, y-\alpha(y))=$ $\left(x-\alpha^{*}(x), y\right)$, which implies $x=\alpha^{*}(x)$. Then

$$
\begin{aligned}
\|x-\alpha(x)\|_{2}^{2} & =\|x\|_{2}^{2}-\left(\alpha^{*}(x), x\right)-\left(x, \alpha^{*}(x)\right)+\|\alpha(x)\|_{2}^{2} \\
& =\|\alpha(x)\|_{2}^{2}-\|x\|_{2}^{2} \leq 0,
\end{aligned}
$$

so $\alpha(x)=x$. By properties of non-commutative $L^{p}$-spaces, the set $X_{0}=$ $H_{0}+i H_{0}$ is dense in $L^{1}$. Moreover, for every $x \in X_{0}, a_{n}(x)$ definitely converges b.a.u. in $\bar{M}$. Consequently, by Theorem 1, we obtain the b.a.u. convergence of $a_{n}(x)$ for every $x \in L^{1}$.

2. Main result. Let $\Omega$ be a compact Hausdorff space, and let $\varphi$ be a continuous map of $\Omega$ into itself such that the family $\left\{\varphi^{n}\right\}_{n \geq 0}$ is equicontinuous. The system $(\Omega, \varphi)$ is called strictly ergodic if there exists a unique $\varphi$-invariant measure $\mu$ on $(\Omega, \mathcal{B})$ with $\operatorname{supp}(\mu)=\Omega$, where $\mathcal{B}$ stands for the $\sigma$-algebra of all Borel subsets of $\Omega$, such that for any $\omega \in \Omega$ and $f \in C(\Omega)$,

$$
\int_{\Omega} f d \mu=\lim _{n \rightarrow \infty} \frac{1}{n} \sum_{k=0}^{n-1} f\left(\varphi^{k} \omega\right)
$$

with respect to the uniform norm in $C(\Omega)$.

Definition 1. A sequence $\left\{k_{i}\right\}$ of non-negative integers is said to be uniform if there exist

(i) a strictly ergodic system $(\Omega, \mathcal{B}, \mu, \varphi)$, 
(ii) a set $Y \in \mathcal{B}$ with $\mu(Y)>0=\mu(\partial Y)$, where $\partial Y$ denotes the boundary of $Y$,

(iii) a point $\omega_{0} \in \Omega$ such that

$$
\begin{aligned}
& k_{1}=\min \left\{k \geq 0: \varphi^{k} \omega_{0} \in Y\right\}, \\
& k_{n}=\min \left\{k>k_{n-1}: \varphi^{k} \omega_{0} \in Y\right\}, \quad n \geq 2 .
\end{aligned}
$$

The triple $(\Omega, \mathcal{B}, \mu, \varphi), Y$ and $\omega_{0}$ is called the apparatus for $\left\{k_{i}\right\}$.

The following two lemmas can be found in [8].

LEMMA 1. If $\left\{k_{i}\right\}$ is a uniform sequence as above, then

$$
\lim _{n \rightarrow \infty} n / k_{n}=\mu(Y) .
$$

LEMma 2. If $\left\{k_{i}\right\}$ is a uniform sequence as above, then for any $\varepsilon>0$ there exist open subsets $Y_{1}, Y_{2}$ and $W$ of $\Omega$ such that

(i) $Y_{1} \subset Y \subset Y_{2}, \mu\left(Y_{2}-Y_{1}\right)<\varepsilon$ and $\mu\left(\partial Y_{1}\right)=0=\mu\left(\partial Y_{2}\right)$,

(ii) $\omega_{0} \in W$ and, for every $\omega \in W$ and all $k \geq 0$,

$$
\chi_{Y_{1}}\left(\varphi^{k} \omega\right) \leq \chi_{Y}\left(\varphi^{k} \omega_{0}\right) \leq \chi_{Y_{2}}\left(\varphi^{k} \omega\right),
$$

where $\chi_{E}(\omega)$ is the characteristic function of a set $E$.

The next simple lemma will be used in the proof of Theorem 4 below.

LEMmA 3. If a sequence $\left\{\widetilde{a}_{n}\right\}$ in $M$ is such that for every $\varepsilon>0$ there are a b.a.u. convergent sequence $\left\{a_{n}\right\} \subset M$ and a positive integer $n_{0}$ satisfying $\left\|\widetilde{a}_{n}-a_{n}\right\|<\varepsilon$ for all $n \geq n_{0}$, then $\left\{\widetilde{a}_{n}\right\}$ converges b.a.u.

Proof. Take arbitrary $\varepsilon, \delta>0$ and find, for every $k$, a sequence $\left\{a_{n}^{(k)}\right\} \subset M$, a projection $p_{k} \in P(M)$ with $\tau\left(p_{k}^{\perp}\right)<\varepsilon / 2^{k}$ and positive integers $n_{0}(k), n_{1}(k)$ such that

$$
\begin{aligned}
\left\|p_{k}\left(a_{m}^{(k)}-a_{n}^{(k)}\right) p_{k}\right\|<\delta / 3 & \forall m, n \geq n_{0}(k), \\
\left\|\widetilde{a}_{n}-a_{n}^{(k)}\right\|<1 / k & \forall n \geq n_{1}(k) .
\end{aligned}
$$

If $p=\bigwedge_{k=1}^{\infty} p_{k}$ then, by the subadditivity of trace, we have $\tau\left(p^{\perp}\right)<\varepsilon$. Moreover, since $p \leq p_{k}$ for all $k$, we obtain, for every $k$,

$$
\left\|p\left(a_{m}^{(k)}-a_{n}^{(k)}\right) p\right\|<\delta / 3
$$

as soon as $m, n \geq n_{0}(k)$. If $k_{0}$ is such that $1 / k_{0}<\delta / 3$, then

$$
\begin{aligned}
\left\|p\left(\widetilde{a}_{m}-\widetilde{a}_{n}\right) p\right\| \leq & \left\|p\left(\widetilde{a}_{m}-a_{m}^{\left(k_{0}\right)}\right) p\right\| \\
& +\left\|p\left(a_{m}^{\left(k_{0}\right)}-a_{n}^{\left(k_{0}\right)}\right) p\right\|+\left\|p\left(\widetilde{a}_{n}-a_{n}^{\left(k_{0}\right)}\right) p\right\|<\delta
\end{aligned}
$$

whenever $m, n \geq \max \left\{n_{0}\left(k_{0}\right), n_{1}\left(k_{0}\right)\right\}$. This means that the sequence $\left\{p \widetilde{a}_{n} p\right\}$ is Cauchy, i.e. $\left\{\widetilde{a}_{n}\right\}$ converges b.a.u.

TheOREM 4. Let $M$ be a vNa with a faithful normal semifinite trace $\tau$, and let $\alpha$ be an absolute contraction on $L^{1}(M, \tau)$. If $\left\{k_{i}\right\}$ is a uniform 
sequence and $x \in L^{1}$, then the averages

$$
\widetilde{a}_{n}(x)=\frac{1}{n} \sum_{i=1}^{n} \alpha^{k_{i}}(x)
$$

converge b.a.u. in $L^{1}$.

Proof. Let $(\Omega, \mathcal{B}, \mu, \varphi)$ and $\omega_{0}, Y$ be the apparatus connected with the sequence $\left\{k_{i}\right\}$. Let $\widetilde{M}$ be the algebra of all essentially bounded ultra-weakly measurable functions $g:(\Omega, \mu) \rightarrow M$ equipped with the trace

$$
\widetilde{\tau}(g)=\int_{\Omega} \tau(g(\omega)) d \mu(\omega),
$$

$g \geq 0$, and let $\widetilde{L}^{1}$ be the Banach space of all Bochner $\mu$-integrable functions $h:(\Omega, \mu) \rightarrow L^{1}(M, \tau)$ (see [7], p. 68). Being the predual to $\widetilde{M}$, the space $\widetilde{L}^{1}$ is isomorphic to $L^{1}(\widetilde{M}, \widetilde{\tau})$. Next, we define a map $\tilde{\alpha}$ on $\widetilde{L}^{1}$ by

$$
\widetilde{\alpha}(h)(\omega)=\alpha(h(\varphi \omega)), \quad h \in \widetilde{L}^{1} .
$$

It can be easily checked that $\widetilde{\alpha}$ is an absolute contraction in $\widetilde{L}^{1}$. Then, by Theorem 2 , we see that, for every $h \in \widetilde{L}^{1}$, the averages

$$
\frac{1}{n} \sum_{k=0}^{n-1} \widetilde{\alpha}^{k}(h)=\frac{1}{n} \sum_{k=0}^{n-1} \alpha^{k}\left(h\left(\varphi^{k}\right)\right)
$$

converge b.a.u. in $\widetilde{L}^{1}$. Repeating the argument of Lemma 2 in [1] shows that the averages

$$
\frac{1}{n} \sum_{k=0}^{n-1} \alpha^{k}\left(h\left(\varphi^{k} \omega\right)\right)
$$

converge b.a.u. in $L^{1}(M, \tau)$ for almost all $\omega \in \Omega$. For an arbitrary $\varepsilon>0$, choose open subsets $Y_{1}, Y_{2}$ and $W$ of $\Omega$ satisfying the conditions of Lemma 2 . Applying the latter convergence to the function $h(\omega)=x \cdot \chi_{Y_{1}}(\omega) \in \widetilde{L}^{1}$ and taking into account that, obviously, $\mu(W)>0$, we find a point $\omega \in W$ for which the sequence

$$
\frac{1}{n} \sum_{k=0}^{n-1} \alpha^{k}(x) \chi_{Y_{1}}\left(\varphi^{k} \omega\right)
$$

converges b.a.u.

On the other hand, since $\mu\left(\partial Y_{i}\right)=0$, we have (see proof of Lemma 1 in $[8])$

$$
\lim _{n \rightarrow \infty} \frac{1}{n} \sum_{k=0}^{n-1} \chi_{Y_{i}}\left(\varphi^{k} \omega\right)=\mu\left(Y_{i}\right), \quad i=1,2
$$


which, together with $\mu\left(Y_{2}\right)-\mu\left(Y_{1}\right)<\varepsilon$ and $\chi_{Y_{1}}\left(\varphi^{k} \omega\right) \leq \chi_{Y}\left(\varphi^{k} \omega_{0}\right) \leq$ $\chi_{Y_{2}}\left(\varphi^{k} \omega\right)$ for every $k$, entails the existence of an $n_{0}$ such that

$$
0 \leq \frac{1}{n} \sum_{k=0}^{n-1}\left[\chi_{Y}\left(\varphi^{k} \omega_{0}\right)-\chi_{Y_{1}}\left(\varphi^{k} \omega\right)\right]<\varepsilon
$$

for all $n \geq n_{0}$. Therefore, for all $n \geq n_{0}$, we have

$$
\begin{aligned}
\| \frac{1}{n} \sum_{k=0}^{n-1} \alpha^{k}(x) \chi_{Y}\left(\varphi^{k} \omega_{0}\right)- & \frac{1}{n} \sum_{k=0}^{n-1} \alpha^{k}(x) \chi_{Y_{1}}\left(\varphi^{k} \omega\right) \| \\
& \leq\|x\| \frac{1}{n} \sum_{k=0}^{n-1}\left[\chi_{Y}\left(\varphi^{k} \omega_{0}\right)-\chi_{Y_{1}}\left(\varphi^{k} \omega\right)\right]<\|x\| \varepsilon .
\end{aligned}
$$

Then Lemma 3 implies that the averages

$$
\frac{1}{n} \sum_{k=0}^{n-1} \alpha^{k}(x) \chi_{Y}\left(\varphi^{k} \omega_{0}\right)
$$

converge b.a.u. Now, since

$$
\begin{aligned}
\frac{1}{n} \sum_{i=1}^{n} \alpha^{k_{i}}(x) & =\frac{k_{n}+1}{n} \cdot \frac{1}{k_{n}+1} \sum_{i=1}^{n} \alpha^{k_{i}}(x) \\
& =\frac{k_{n}+1}{n} \cdot \frac{1}{n} \sum_{k=0}^{n-1} \alpha^{k}(x) \chi_{Y}\left(\varphi^{k} \omega_{0}\right),
\end{aligned}
$$

by Lemma 1 , we obtain the b.a.u. convergence of the averages $\widetilde{a}_{n}(x)$ for every $x \in L^{1}(M, \tau) \cap M$.

Finally, if $x \in L^{1}, x \geq 0$, then, according to Theorem 3 , given $\varepsilon>0$, one finds $e \in P(M)$ with $\tau\left(e^{\perp}\right) \leq \varepsilon$ such that

$$
\sup _{n}\left\|e a_{n}(x) e\right\|<\infty .
$$

Therefore we also have

$$
\sup _{n}\left\|e \widetilde{a}_{n}(x) e\right\| \leq\left[\sup _{n} \frac{k_{n}+1}{n}\right] \cdot\left[\sup _{n}\left\|e a_{n}(x) e\right\|\right]<\infty,
$$

and then

$$
\sup _{n}\left\|\widetilde{a}_{n}(x) e\right\|<\infty \quad \text { for all } x \in L^{1} .
$$

Hence, by Theorem 1, and taking into account that $X_{0}=L^{1} \cap M$ is dense in $X=L^{1}$, we get the result. 


\section{References}

[1] N. Dang-Ngoc, A random ergodic theorem in von Neumann algebras, Proc. Amer. Math. Soc. 86 (1982), 605-608.

[2] T. Fack and H. Kosaki, Generalized s-numbers of $\tau$-measurable operators, Pacific J. Math. 123 (1986), 269-300.

[3] M. Goldstein and S. Litvinov, Banach principle in the space of $\tau$-measurable operators, Studia Math. 143 (2000), 33-41.

[4] R. V. Kadison, A generalized Schwarz inequality and algebraic invariants for operator algebras, Ann. of Math. 56 (1952), 494-503.

[5] E. C. Lance, Noncommutative ergodic theorems, in: Proc. of the Meeting on $C^{*}$ algebras and their Appl. to Theor. Phys. (Roma, 1975), Academic Press, London, 1976, 39-47.

[6] E. Nelson, Notes on non-commutative integration, J. Funct. Anal. 15 (1974), 103116.

[7] S. Sakai, $C^{*}$-algebras and $W^{*}$-algebras, Springer, Berlin, 1971.

[8] R. Sato, Operator averages for subsequences, Math. J. Okayama Univ. 22 (1980), 161-168.

[9] I. Segal, A non-commutative extension of abstract integration, Ann. of Math. 57 (1953), 401-457.

[10] M. Takesaki, Theory of Operator Algebras I, Springer, Berlin, 1979.

[11] F. J. Yeadon, Ergodic theorems for semifinite von Neumann algebras I, J. London Math. Soc. (2) 16 (1977), 326-332.

Department of Mathematics

North Dakota State University

Fargo, ND 58105, U.S.A.

Current address:

Department of Statistics

St. Cloud State University

St. Cloud, MN 56301, U.S.A.

E-mail: slitvinov@stcloudstate.edu
Institute of Mathematics Uzbekistan Academy of Sciences F. Hodjaev st. 29 Tashkent, 700143, Uzbekistan E-mail: tmf000@tashsu.silk.org 